\title{
Soluble InP and GaP Nanowires: Self-Seeded, Solution-Liquid-Solid Synthesis and Electrical Properties
}

Zhaoping Liu, ${ }^{[a]}$ Kai Sun, ${ }^{[b]}$ Wen-Bin Jian,,${ }^{[c]}$ Dan Xu,${ }^{[a]}$ Yen-Fu Lin,${ }^{[c]}$ and Jiye Fang ${ }^{*[a]}$

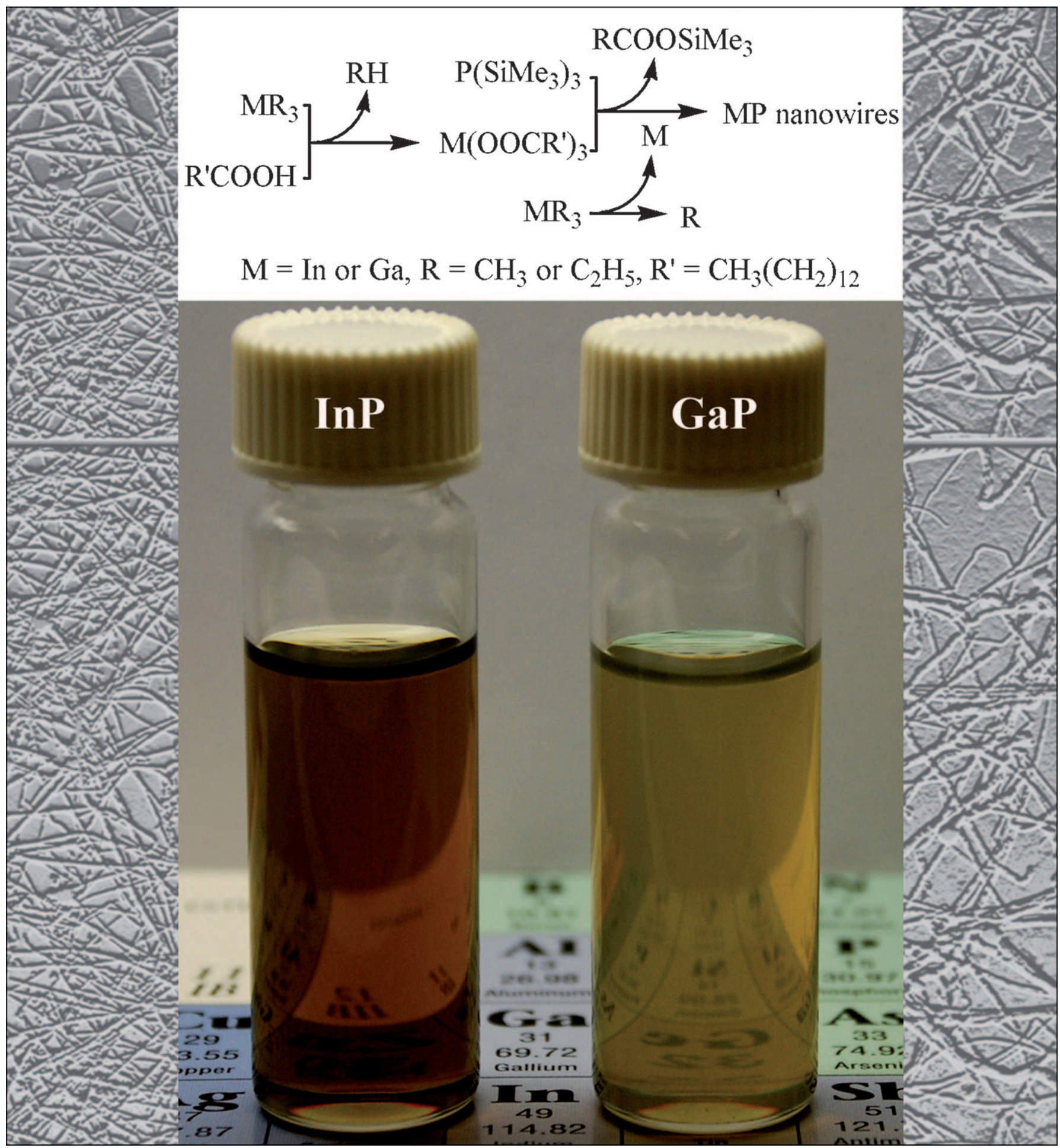




\begin{abstract}
We demonstrate a facile method for self-seeded, solutionliquid-solid growth of soluble InP and $\mathrm{GaP}$ nanowires at a temperature of $\approx 300^{\circ} \mathrm{C}$. Both types of nanowires are single crystals with very small diameters. The synthesized InP nanowires are almost defect-free, whereas the $\mathrm{GaP}$ nanowires have some microtwins. The effect of reaction temperatures and input ligand/III/V (III and V indi-
\end{abstract}

cate elements of Group 13 and 15 respectively) ratios on wire formation is discussed, and two competitive chemical pathways involved in the nanowire formation are proposed. In addition, electrical properties of these III-V

Keywords: electrical properties gallium • indium • nanowires phosphorus $\cdot$ semiconductors nanowires, generated from the solution-based approach, were investigated for the first time. The current-voltage $(I-V)$ and room temperature resistance investigations indicate that both InP and $\mathrm{GaP}$ nanowires possess very low native point defects for carrier concentrations and they could be potentially promising building blocks in optoelectronic applications.

\section{Introduction}

The synthesis of Group 13-Group 15 semiconductor nanowires (III-V NWs) has been a subject of intensive studies during the past two decades, because these NWs offer attractive applications in nano-optics and nanoelectronics. ${ }^{[1-3]}$ Highly crystalline III-V NWs have been successfully synthesized by various vapor-based techniques, such as vaporliquid-solid (VLS) growth, ${ }^{[4-7]}$ laser-assisted catalytic growth, ${ }^{[8,9]}$ oxide-assisted growth, ${ }^{[10]}$ template-induced growth, ${ }^{[11,12]}$ and thermal evaporation growth. ${ }^{[13]}$ However, due to the high temperature processes, the NWs grown in vapors generally have diameters on the order of tens of nanometers, which are much larger than the bulk exciton Bohr radius of III-V semiconductors. Consequently, the IIIV NWs obtained by these methods show no quantum-confinement effects. The preparation of small diameter NWs has been explored through various solution-based syntheses. ${ }^{[14]}$ In 1995, Buhro and co-workers first prepared one-dimensional structures of InP, InAs, and GaAs through solution-phase reactions at relatively low temperatures $\left(\approx 200^{\circ} \mathrm{C}\right)$ in the presence of low-melting-point metal In or alloys In/Ga. ${ }^{[15]}$ They proposed a solution-liquid-solid (SLS) mechanism, as an analogy to the VLS mechanism, for the catalyzed growth of these anisotropic nanostructures. Through their continuous efforts, they improved the SLS method and produced III-V as well as other semiconductor

[a] Z. Liu, D. Xu, Prof. Dr. J. Fang

Department of Chemistry

State University of New York at Binghamton

Binghamton, New York 13902 (USA)

Fax: (+1) 6077774478

E-mail: jfang@binghamton.edu

[b] Dr. K. Sun

Department of Materials Science and Engineering

University of Michigan

Ann Arbor, Michigan 48109 (USA)

[c] Prof. Dr. W.-B. Jian, Y.-F. Lin

Department of Electrophysics

National Chiao Tung University Hsinchu, 30010 (Taiwan)

Supporting information for this article is available on the WWW under http://dx.doi.org/10.1002/chem.200900190.
NWs with high crystallinity and controlled diameters using monodisperse nanoparticles (NPs) of low-melting-point metals (In or $\mathrm{Bi}$ ) as the catalytic seeds. ${ }^{[16-22]}$ Concurrently, Korgel and co-workers ${ }^{[23,24]}$ successfully extended their supercritical fluid-liquid-solid NW growth approach to GaP and GaAs NWs by using Au nanocrystal seeds. Nevertheless, a high temperature $\left(\approx 500^{\circ} \mathrm{C}\right)$ and high pressure $(37 \mathrm{MPa})$ are required in this special reaction setup.

Because a surfactant is involved in the SLS growth, it appears that this is one of a few methods that offer soluble III-V NWs in solution. However, pre-prepared In or Bi NP seeds are required for this strategy. Although Bi NPs are readily available, their high surface activity ${ }^{[25]}$ and direct incorporation to the NWs as an heterogeneous phase could be an issue in optoelectronic applications. ${ }^{[19,20]}$ Compared with $\mathrm{Bi}$ NPs, with the exception of the heterogeneity issue for GaP NW preparation, the synthesis and storage of In NPs are much more difficult due to their higher reactivity. Although colloidal $\mathrm{Ga}$ is a good candidate as the catalyst, $\mathrm{Ga}$ NPs are unavailable to date. It is hence a challenge to further refine the syntheses of In-V/Ga-V NWs on the basis of SLS approach by using a constituent catalyst, that is, In or Ga NPs.

Most recently, Burho and co-workers ${ }^{[26]}$ improved this SLS strategy and demonstrated a self-catalyzed SLS growth of GaAs NWs from Ga in-situ droplets provided by a decomposition of tributylgallium $\left(\mathrm{Ga}(n \mathrm{Bu})_{3}\right)$. In their synthesis, the success is strongly dependent on a polymeric solvent and a home-made, uncommon copolymer surfactant. In the present work, a more facile route to fabricate InP and $\mathrm{GaP}$ NWs has been developed. In this simplified preparation, commercially available In/Ga precursors and more common solvents/surfactants are employed. The synthesis was carried out in an organic solution by using 1-octadecene (ODE) as the solvent, myristic acid as the capping ligand (surfactant), tris(trimethylsilyl)phosphine $\left(\mathrm{P}\left(\mathrm{SiMe}_{3}\right)_{3}\right)$ as the $\mathrm{P}$ source, trimethylindium $\left(\mathrm{InMe}_{3}\right)$ or triethylgallium $\left(\mathrm{GaEt}_{3}\right)$ as the metal source and the in-situ catalyst seeds in form of In/Ga droplets. An investigation of electronic transport properties on these NWs is also presented. 


\section{Results and Discussion}

Characteristics of reaction products: The InP or GaP NWs were quickly generated as a flocculent precipitate upon injecting the mixed solution of $\mathrm{P}(\mathrm{SiMe} 3) 3$ and $\mathrm{InMe} 3$ (or GaEt3) into a hot ODE containing myristc acid. As a byproduct, the reaction also yielded a small amount of colloidal InP (or GaP) NPs, indicated by the reddish (or pale yellow) color of the supernatant solution. Fortunately, the NPs remaining in the NW precipitates could be completely removed by multiple cycles of washing with hexane and centrifugation, resulting in pure NWs. From typical syntheses, the yields of both InP and GaP NWs were estimated to be $\approx 90 \%$ on the basis of $\mathrm{P}(\mathrm{SiMe} 3) 3$. The NW products, which were initially precipitated as tangled floccules, are readily redispersed in toluene through ultrasonication, forming a translucent suspension. The dilute suspension of InP NWs is light brown, and that of $\mathrm{GaP}$ is greenish yellow (shown in the frontispiece). They were very stable and no evident sediment was determined upon standing for weeks. This character indicates that the as-prepared NWs were surface-capped by surfactant because of the high stability. Such excellent solubility in organic solvents benefits to various NW characterizations and related applications.

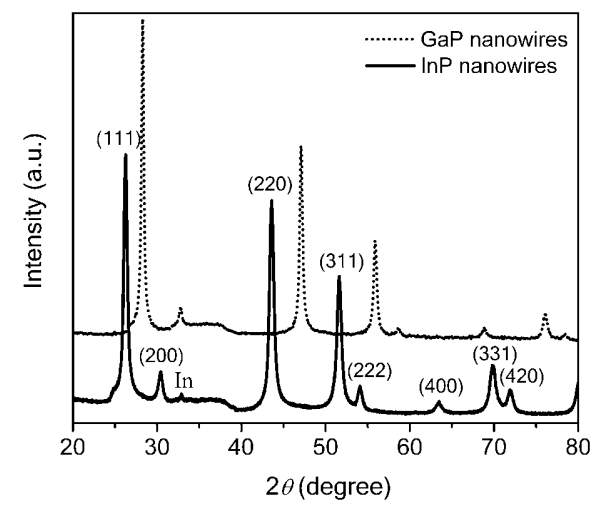

Figure 1. XRD patterns of InP (bottom, solid line) and GaP (top, dot line) nanowires.

Crystal structure, morphology and microstructure of nanowires: Figure 1 shows XRD patterns of the typical InP and GP NWs. The sharp reflection peaks are indexed as those from standard diffraction cards of InP (refer to JCPDS card 32-0452) and GaP (JCPDS card 32-0397), indicating their single-phase zinc blende (or sphalerite) structure (space group $F \overline{4} 3 m(216)$ ). The lattice constants calculated from both patterns, $a=0.586 \mathrm{~nm}$ for the InP sample and $0.545 \mathrm{~nm}$ for $\mathrm{GaP}$, are consistent with the standard values in the JCPDS cards, respectively. The high intensity of those peaks implies the presence of high crystallinity in the products. In both XRD patterns, there is a broad weak reflection around $37^{\circ}$, which may be assigned to a trace of amorphous In or $\mathrm{Ga}$. In addition, a weak peak corresponding to the (101) diffraction peak of In can be detected in the pattern of InP.
Figure 2 presents typical transmission electron microscopy (TEM) images of the InP and GaP NWs. As shown, both samples are dominated by somewhat kinky NWs with lengths of several micrometers. The InP NWs have diame-
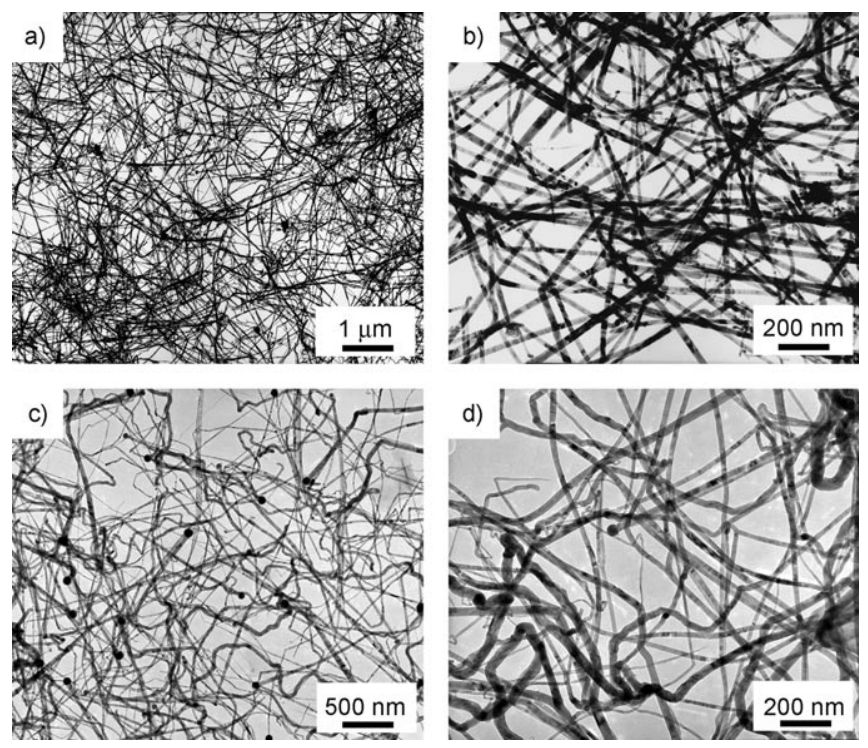

Figure 2. Low- and high-magnification TEM images of the nanowire samples: a,b) InP and c,d) GaP.

ters ranging from 10 to $30 \mathrm{~nm}$ with an average value of approximately $20 \mathrm{~nm}$, whereas the GaP NWs generally possess diameters varying from 5 to $50 \mathrm{~nm}$. We surmise that the relatively wide diameter distribution for GaP NWs may be due to the easy-aggregation nature of liquefied Ga NPs. The GaP NW usually has a droplet at the tip, showing the distinct character of SLS growth. In contrast, most of the InP NWs do not have tip droplets, which might have been consumed at the end stage of InP NW growth.

High-resolution transmission electron microscopy (HRTEM) images and selected-area electron diffraction (SAED) patterns reveal that both InP and GaP NWs exhibit zinc blende structure with a preferential orientation along $\langle 111\rangle$ direction. Figure $3 a$ and $b$ depicts typical HRTEM images of a single InP and GaP NW, respectively, and their corresponding SAED patterns (insets). The HRTEM images
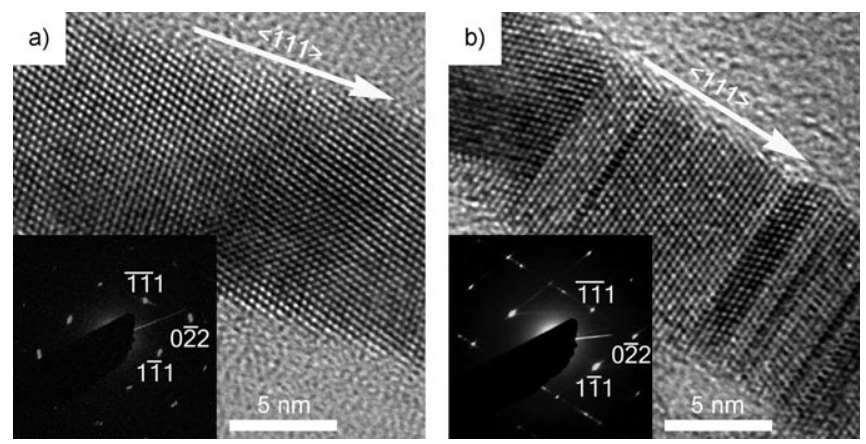

Figure 3. HRTEM images of individual nanowires: a) InP and b) GaP. 
show not only that the single-crystal NWs of both InP and $\mathrm{GaP}$ anisotropically grow along a $\langle 111\rangle$ direction, but they also reveal a fact that the lattice spacing distances $(0.34 \mathrm{~nm}$ for $\mathrm{InP}$ and $0.31 \mathrm{~nm}$ for $\mathrm{GaP}$ ) measured from the lattice fringes match their interplanar spacings of adjacent $\{111\}$ lattice planes well. The SAED patterns of the InP and GaP single NWs presented in the insets of Figure $3 a$ and $b$, respectively, further confirm this attribution (image projection direction: $\langle 011\rangle)$. Additional HRTEM observations indicate that the InP NWs are almost defect-free single crystals, whereas the GaP NWs have a considerable amount of twining faults. The twins are confined to $\{111\}$ planes and distributed randomly along the length of the NW. The twin faults may be responsible for the kinky morphology for the GaP NWs, as demonstrated elsewhere. ${ }^{[27]}$ In addition, energy-dispersive X-ray spectrometry (EDS) analyses (see the Supporting Information, Figure S1) suggest that the central sections of the NWs are qualified as stoichiometric InP (or $\mathrm{GaP}$ ), whereas the tip droplets are indexed as almost the pure metal In (or Ga). These results support our postulated mechanism that the NW growth was indeed catalyzed by the In or Ga droplets.

Effect of reaction temperature: In our synthetic strategy, $\mathrm{InMe}_{3}$ and $\mathrm{GaEt}_{3}$ were used as the sources of Group 13 elements as well as the precursors of catalytic seeds. The decomposition temperature of these metal-organic precursors is close to $300^{\circ} \mathrm{C},{ }^{[28-30]}$ enabling seed-generation from their decomposition prior to NW formation. The temperatures of $290^{\circ} \mathrm{C}$ for $\mathrm{InP}$ and $300^{\circ} \mathrm{C}$ for $\mathrm{GaP}$, applied to the typical syntheses, have been determined to be the optimized seed-generation conditions. In principle, a decrease of temperature is associated with a lower decomposition efficiency, and may lead to insufficient or even no seeds. As a result, a low yield or no NW could be produced. In the InP system, for instance, a reaction at $265^{\circ} \mathrm{C}$ only yielded aggregates of InP $\mathrm{NPs}$; at $270^{\circ} \mathrm{C}$, it gave NWs combined with InP NPs (Figure $4 \mathrm{a}$ ); up to $280^{\circ} \mathrm{C}$, it generated a product of pristine NWs (Figure 4b). Despite the impurity, NWs grown at lower temperatures showed relatively smaller diameters. The average diameters for InP NWs synthesized at 280 and $270^{\circ} \mathrm{C}$ were approximately 17 and $14 \mathrm{~nm}$, respectively, which are a bit smaller than the value $(20 \mathrm{~nm})$ of those prepared at $290^{\circ} \mathrm{C}$. This indicates a weak influence of the temperature range between 270 and $290^{\circ} \mathrm{C}$ on the average di-

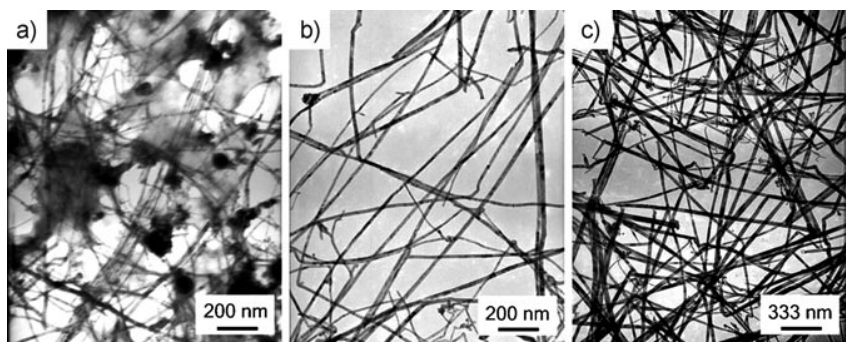

Figure 4. TEM images of InP samples prepared at different temperatures: a) $270{ }^{\circ} \mathrm{C}$, b) $280^{\circ} \mathrm{C}$, and c) $300^{\circ} \mathrm{C}$. ameter of InP NWs. When the reaction temperature is higher than $290^{\circ} \mathrm{C}$, however, the influence of the temperature becomes more prominent. Particularly, the InP NWs grown at $300{ }^{\circ} \mathrm{C}$ had an average diameter as large as $30 \mathrm{~nm}$ (Figure $4 \mathrm{c}$ ). This is likely due to more intense coalescence between the liquefied seeds/droplets.

Effect of MA/III/V ratio (MA = myristic acid): It was realized that the molar ratio of myristic acid, $\mathrm{InMe}_{3}$ (or $\mathrm{GaEt}_{3}$ ), and $\mathrm{P}\left(\mathrm{SiMe}_{3}\right)_{3}$ (designated as $\mathrm{MA} / \mathrm{III} / \mathrm{V}$ ) plays an important role in the control of NW yield and purity. For the InP NW synthesis, the MA/In/P ratio was optimized at $3: 1.5: 1$. At given dosages of the In and $\mathrm{P}$ precursors, an increase of the $\mathrm{MA} / \mathrm{In}$ ratio resulted in a reduced yield of InP NWs as well as an increasing output of InP NPs. This tendency was evidenced from the fact that a smaller amount of floccules and a deeper red color of the supernatant solution was observed when the MA/In ratio was increased. When the MA/In ratio reached $4: 1.5$, the reaction yielded a transparent dark red solution that contained colloidal InP NPs only. Actually, this condition is close to that for the preparation of InP NPs with a MA/In ratio of $4: 1$ reported by $\mathrm{Xu}$ et al. ${ }^{[31]}$ This implies that $\mathrm{InMe}_{3}$ does not decompose to seeds when an excess of myristic acid is used. In contrast, applying a MA/ In ratio of $<3: 1.5$ introduces an "impurity" of In NPs, and the amount of In NPs increases as the MA/In ratio further decreases. For instance, the NWs obtained at MA/In of 2:1 were in company with a considerable amount of In aggregates with tens of nanometers in size (Figure $5 \mathrm{a}$ ). When no
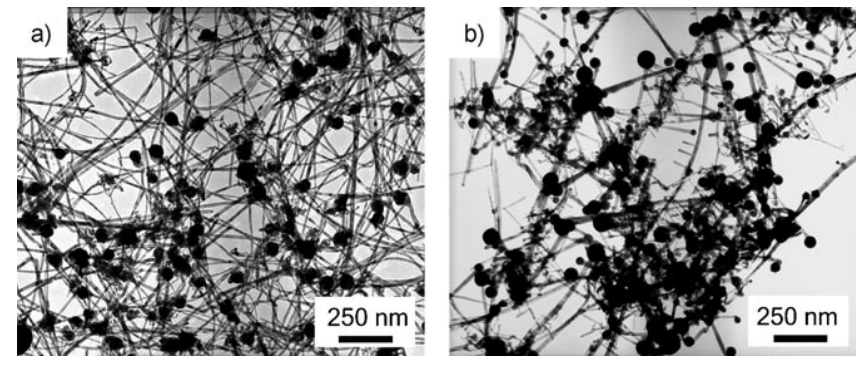

Figure 5. TEM images of InP samples prepared a) at $\mathrm{MA} / \mathrm{In} / \mathrm{P}=2: 1.5: 1$, and $b$ ) without myristic acid

myristic acid was applied to the synthesis, In NPs became the major product, although a considerable amount of NWs also formed (Figure 5b). Nevertheless, the NWs grown under this condition exhibited an abundant amount of short branches. The formation of branched NWs is likely due to the Ostwald ripening growth on the previously formed NWs.

Additionally, the variation of $\mathrm{In} / \mathrm{P}$ ratios was also able to affect the NW purity, indicating an opposite effect of MA/In ratios. An In/P ratio of $>1.5: 1$ generally yields aggregations of In NPs as a heterophase, whereas an In/P ratio of $<1.5: 1$ results in discrete InP NPs as "impurity". These experiments indicate that the 0.5 -fold excess of $\mathrm{InMe}_{3}$ with respect to P$\left(\mathrm{SiMe}_{3}\right)_{3}$ is exactly able to provide sufficient, but no excess, of In seeds for the NW formation under the present synthet- 
ic conditions. Since it has been demonstrated that the SLS growth of III-V NWs requires a molar ratio of group 13 (or 15) precursors/seeds of at least ten ${ }^{[19,26,32]}$ we believe that most of the excessive $\mathrm{InMe}_{3}$ (possibly more than $80 \%$ of the excess) might be consumed through a volatilization in the SLS process.

In comparison with the InP NW preparation, it was determined that for the $\mathrm{GaP}$ formation an excess amount of $\mathrm{GaEt}_{3}$ was required with an optimized $\mathrm{MA} / \mathrm{Ga} / \mathrm{P}$ ratio at $3: 9: 1$. The reason may lie in the fact that $\mathrm{GaEt}_{3}$ is more stable than $\mathrm{InMe}_{3}$, as the bonding energy of $\mathrm{Ga}-\mathrm{C}$ $\left(\approx 56.7-57.7 \mathrm{kcal} \mathrm{mol}^{-1}\right)^{[33-35]}$ is larger than that of $\mathrm{In}-\mathrm{C}$ $\left(\approx 38.9 \mathrm{kcal} \mathrm{mol}^{-1}\right) \cdot{ }^{[28,36]}$ Therefore, an appropriately high concentration of $\mathrm{GaEt}_{3}$ is essential to kinetically achieve the equilibrium and to enable the relevant decomposition. Nevertheless, the MA/Ga and $\mathrm{Ga} / \mathrm{P}$ ratios showed the same effect as that exhibited in the InP NW synthesis.

Chemical pathway of the nanowire formation: Upon understanding the effects of reaction temperature and MA/III/V ratios, we envisage that the reaction between $\mathrm{InMe}_{3}$ (or $\mathrm{GaEt}_{3}$ ) and myristic acid must take place prior to all other possible reactions immediately after an injection of the In (or Ga) and $\mathrm{P}$ precursors. This reaction yields indium (or gallium) myristate that is more reactive with $\mathrm{P}\left(\mathrm{SiMe}_{3}\right)_{3}$ than the alkyl-metal compounds. The reaction between the myristate and $\mathrm{P}\left(\mathrm{SiMe}_{3}\right)_{3}$ quickly generates amorphous $\mathrm{InP}$ (or $\mathrm{GaP}$ ) clusters. As a parallel reaction pathway, the remaining $\mathrm{InMe}_{3}$ (or $\mathrm{GaEt}_{3}$ ) molecules simultaneously decompose into In (or $\mathrm{Ga}$ ) clusters through an elimination of methyl (or ethyl) radicals. Once metal In (or Ga) droplets come into being, the SLS NW growth occurs naturally with a dissolution of the III-V clusters into the liquefied metal droplets. These processes towards the NW formation as two chemical pathways are summarized in Scheme 1 . We therefore con-

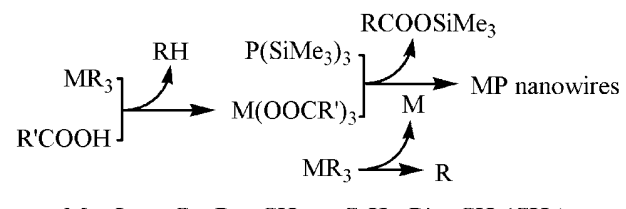

$\mathrm{M}=$ In or $\mathrm{Ga}, \mathrm{R}=\mathrm{CH}_{3}$ or $\mathrm{C}_{2} \mathrm{H}_{5}, \mathrm{R}^{\prime}=\mathrm{CH}_{3}\left(\mathrm{CH}_{2}\right)_{12}$

Scheme 1. Proposed chemical pathway for the nanowire formation.

clude that a success of the III-V NW formation is dependent on a competition of both above-mentioned reactions, which are controlled by the concentration of the In (or $\mathrm{Ga}$ ) precursors, myristic acid, and $\mathrm{P}$ precursors. Though the NW formation involves a series of complex chemical reactions, the growth process should be quite fast. Controlled experiments, in which the reaction was quenched by an addition of cold ODE, revealed that a nearly pure NW product could form within one minute (Supporting Information, Figure S2).
Electrical properties of nanowires: The excellent solubility and the micrometer-scale length of these NWs facilitate the measurement of their electric properties. For this purpose, the InP NWs were selected as a model system, owing to their fewer defects, and two nanocontact InP NW devices were fabricated for electrical characterization. ${ }^{[37]}$ A typical field-emission scanning electron microscope (FE-SEM) image of two Ti/Au electrodes contacted on an InP NW is shown in Figure $6 \mathrm{a}$. The distance separating the contacted
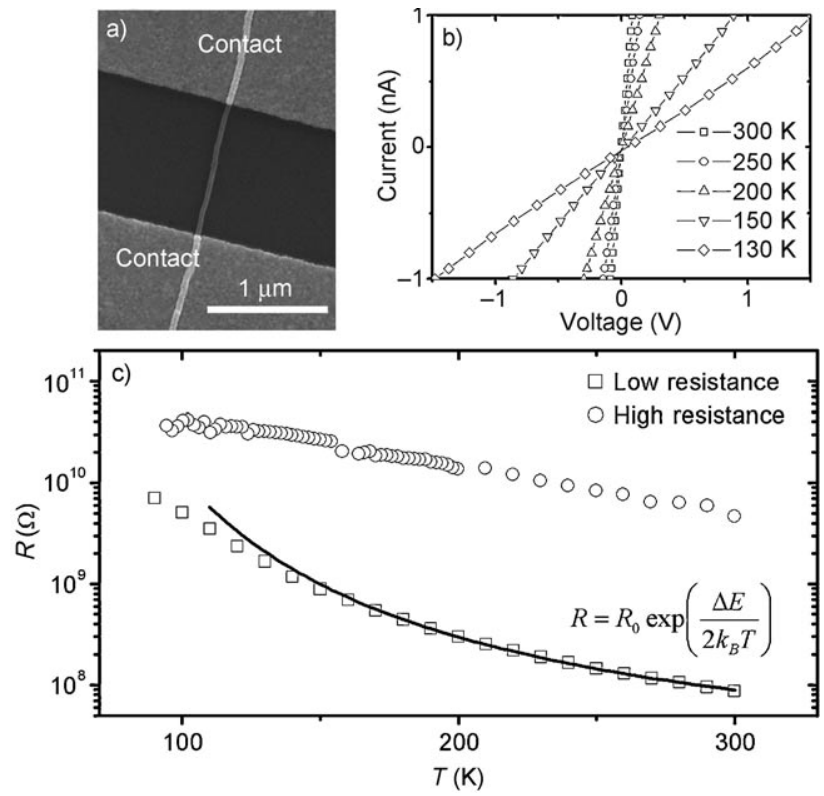

Figure 6. a) Typical FE-SEM image of InP nanowire devices with a diameter of $\approx 25 \mathrm{~nm}$ and a two-contact configuration. b) Temperature-dependent $I-V$ curves of the nanowire device with a RT resistance of $\approx 87 \mathrm{M} \Omega$. c) Temperature-dependent resistances in the two typical devices with lower (squares) and higher (circles) RT resistances. The solid line gives the best fit to thermally activated transport of the displayed equation.

electrodes was kept constant for all NW devices. The devices with a low resistance of about $100 \mathrm{M} \Omega$ at room-temperature (RT) had a noticeable linear current-voltage $(I-V)$ behavior between -1.5 and $1.5 \mathrm{~V}$ at various temperatures down to $130 \mathrm{~K}$ (Figure $6 \mathrm{~b}$ ). The linear $I-V$ relationship supports our conjecture of two Ohmic contacts on an individual InP NW for low-resistance RT devices. To further corroborate this, electron transport in devices with low and high resistances at RT was investigated and is depicted in Figure $6 \mathrm{c}$. The low-resistance device can be well described by the thermally activated transport theory of electrons in semiconductors, whereas the high-resistance device, with a resistance value 100 times larger than that the low-resistance device at RT, demonstrates a resistance dominated by the two contact electrodes and cannot be fitted according to the thermally activated transport theory. ${ }^{[37,38]}$ We therefore believe that our low-resistance device gives intrinsic electrical properties of the InP NW, and the resistivity at RT was estimated to be $\approx 10 \Omega \mathrm{cm}$. Since only few structural defects were observed in Figure $3 \mathrm{a}$ and the InP NWs were almost straight (Fig- 
ure $2 \mathrm{a}$ and $\mathrm{b}$ and Figure $6 \mathrm{a}$ ), we can assume that the mobility of carriers in the InP NWs should have the same magnitude of $\approx 1000 \mathrm{~cm}^{2} \mathrm{~V}^{-1} \mathrm{~s}$ as that in a bulk. ${ }^{[20]}$ According to previous studies, the carrier concentration of such InP NW can be estimated to be about $10^{15} \mathrm{~cm}^{-3}$, indicating $\approx 1000$ times fewer defects (carrier concentration) than others reported in previous literature. ${ }^{[39,40]}$ Such a low carrier concentration not only confirms that there are fewer native defects in our InP NWs but also indicates that they could potentially be beneficial in optoelectronic applications.

The electrical properties of $\mathrm{GaP} \mathrm{NWs}^{[41]}$ were also evaluated and their results are presented in Figure S3 in the Supporting Information. Figure $\mathrm{S} 3$ a gives $I-V$ dependence of seven GaP NW devices with two contact electrodes (shown in the inset). A linear $I-V$ behavior with a wide range of voltage was determined, which suggests that Ohmic contacts exist in these $\mathrm{GaP}$ devices. To confirm this supposition, temperature behavior of $I-V$ curves for the GaP-5 device was further investigated and is shown in Figure S3b with a corresponding resistance-temperature curve in the inset. As expected, the temperature-dependent resistance can be fitted in agreement with thermally activated transport theory. Since the device resistance is dominated by the GaP NWs, we can estimate the NW resistivity to be $\approx 500 \Omega \mathrm{cm}$ and the carrier concentration to be $\approx 10^{14} \mathrm{~cm}^{-3}$ by using the bulk mobility of $160 \mathrm{~cm}^{2} \mathrm{~V}^{-1} \mathrm{~s} .{ }^{[42]}$ The electrical characteristics of GaP NWs suggest that fewer native point defects and carrier concentration exist in these GaP NWs. Compared with InP NWs, we realize that the carrier concentrations in GaP NWs approaches the same magnitude, that is, $10^{14}-10^{15} \mathrm{~cm}^{-3}$. The relatively low carrier concentrations in both $\mathrm{InP}$ and $\mathrm{GaP}$ NWs imply the same generation route of the native point defects from this self-seeded SLS synthetic approach. We therefore believe that this as-developed strategy can provide a NW with few intrinsic carriers as well as few point defects.

\section{Conclusions}

In summary, a self-seeded SLS method has been successfully modified for the synthesis of soluble InP and GaP NWs. In addition to the InP (or GaP) formation through a combination between myristate and $\mathrm{P}\left(\mathrm{SiMe}_{3}\right)_{3}$, the catalyst seeds were in-situ and competitively generated by a decomposition of the metal-organic precursors, $\mathrm{InMe}_{3}$ or $\mathrm{GaEt}_{3}$, at an appropriately high temperature. The reaction temperature and the molar ratio of ligand/III/V (i.e., MA/In/P or MA/ $\mathrm{Ga} / \mathrm{P}$ ) were realized to be the crucial factors for the NW formation. This method is relatively facile as the solvent and surfactant used are common and no additional catalyst seeds are required. Since the NW growth precludes a second-phase catalyst, a possible heterogeneous contamination on NWs is avoided.

The microstructure of the resultant III-V NWs, rationally explored using an HRTEM, shows a high-quality of InP NWs that are almost defect-free single crystals, whereas the GaP NWs have a considerable amount of $\{111\}$-plane-con- fined twining faults. The electrical properties of these selfseeded SLS-generated NWs were also studied for the first time. The exploration of the $I-V$ relationship and RT resistance indicates that both $\mathrm{InP}$ and GaP NWs possess very low native defects for carrier concentration and that they could be potentially promising in optoelectronic applications.

Furthermore, given the active sources of other Group 15 elements, that is, tris(trimethylsilyl)arsine $\left(\mathrm{As}\left(\mathrm{SiMe}_{3}\right)_{3}\right)$ and tris(tri-methylsilyl)stibine $\left(\mathrm{Sb}\left(\mathrm{SiMe}_{3}\right)_{3}\right)$ available, this novel synthetic strategy can be readily extended to a preparation of other III-V NWs including GaAs, GaSb, InAs, InSb, and their $(\mathrm{Ga} / \mathrm{In})(\mathrm{P} / \mathrm{As} / \mathrm{Sb})$ alloys. Although the self-seeded method exploited in this work creates an alternative solution-based approach of producing high-quality III-V NWs, improvement on further reducing the average diameter of these NWs to the level of their exciton Bohr radii is still essential and expected in future work.

\section{Experimental Section}

Chemicals: All reagents were of analytical or better grade and were used as received from Aldrich (except $\operatorname{InMe}_{3}(99.9 \%)$ and $\mathrm{Ga}\left(\mathrm{C}_{2} \mathrm{H}_{6}\right)_{3}(97 \%)$ from STREM, and $\mathrm{P}\left(\mathrm{SiMe}_{3}\right)_{3}$ from Alfa Aesar) without further purification.

Nanowire syntheses: The syntheses were conducted using a Schlenk line technique. For a typical synthesis of InP NWs, ODE $(90 \%, 10 \mathrm{~mL})$ containing myristic acid $(99 \%, 0.3 \mathrm{mmol})$ in a three-neck flask equipped with a condenser was heated to $120^{\circ} \mathrm{C}$ and pumped for $30 \mathrm{~min}$ using a mechanical vacuum pump. The system was purged and then heated up to $305^{\circ} \mathrm{C}$ under $\mathrm{Ar}$ stream. Subsequently, a solution of $\mathrm{P}\left(\mathrm{SiMe}_{3}\right)_{3}$ $(0.1 \mathrm{mmol}), \mathrm{InMe}_{3}(0.15 \mathrm{mmol})$, and trioctylphosphine (TOP, $90 \%$, $0.15 \mathrm{mmol})$ in ODE $(2 \mathrm{~mL})$, which was pre-prepared in a glove box filled with $\mathrm{Ar},{ }^{[43]}$ was swiftly injected into the flask under a vigorous stirring. Addition of the mixed solution decreased the temperature to $290^{\circ} \mathrm{C}$, at which point the reaction was allowed to proceed for $5 \mathrm{~min}$ before the heating source was quickly removed. The reaction resulted in a blackbrown flocculent precipitate and a reddish solution. The precipitate was collected by centrifugation and washed with hexane for several cycles.

The synthesis of GaP NWs was carried out using a similar procedure mentioned above. Typically, a solution of $\mathrm{P}\left(\mathrm{SiMe}_{3}\right)_{3}(0.1 \mathrm{mmol})$ and $\mathrm{GaEt}_{3}(0.9 \mathrm{mmol})$ in ODE $(2 \mathrm{~mL})$ was injected into a pre-heated solution of myristic acid $(0.3 \mathrm{mmol})$ in $\mathrm{ODE}(10 \mathrm{~mL})$ at $315^{\circ} \mathrm{C}$. After the addition, the reaction temperature rapidly dropped to $300^{\circ} \mathrm{C}$. The reaction was allowed to settle for $5 \mathrm{~min}$, yielding a greenish grey flocculent precipitate and a yellowish solution.

Characterization: X-ray diffraction (XRD) data of the powder samples was collected by using a PANalytical $\mathrm{X}^{\prime}$ Pert system equipped with a $\mathrm{Cu}_{\mathrm{K} a}$ radiation source $(\lambda=0.15406 \mathrm{~nm})$. A Hitachi 7000 transmission electron microscope (TEM) operated at $110 \mathrm{kV}$ was used for conventional TEM imaging, and a JEOL-2010 FEG TEM operated at $200 \mathrm{kV}$ was employed for high-resolution TEM imaging (HRTEM), selected-area electron diffraction (SAED), and X-ray energy-dispersive spectroscopic (EDS) data collection.

Electrical property measurement: NW devices were fabricated by using the following method: A silicon substrate was capped with a $400 \mathrm{~nm} \mathrm{SiO}_{2}$ layer to prevent current leakage through the substrate and it was then patterned with gold micron electrodes through a standard lithography procedure. A drop of toluene suspension containing dispersed NWs was subsequently deposited on the silicon substrate. The as-deposited NWs were randomly distributed and a selected NW was positioned using a field-emission scanning electron microscope (FE-SEM, JEOL JSM7000F). A standard e-beam lithography and thermal evaporation method was then employed to fabricate two submicron Ti/Au (40/200 nm in thick- 
ness) electrodes on the selected individual NW in connection with gold micron electrodes, and the separation distance between the two electrodes on the NW was kept at a constant length of $\approx 1 \mu \mathrm{m}$. Finally, the asfabricated NW devices were heated at $400^{\circ} \mathrm{C}$ in a high vacuum for several minutes to improve their electrical contact before they were placed in a liquid-nitrogen cryostat for the temperature dependent evaluation. The current-voltage $(I-\mathrm{V})$ measurements were carried out with a current resolution of $10 \mathrm{pA}$ and a voltage resolution of $1 \mathrm{mV}$. The error in the resistance estimation was less than $0.1 \%$. The settling time for each $I-V$ data point was $10 \mathrm{~s}$, and no perceptible hysteresis loops were observed during our measurements. In particular, several weeks later, the same $I-V$ curves were obtained in the same NW device.

\section{Acknowledgements}

This work was supported by NSF CAREER program (DMR-0731382) and by State University of New York at Binghamton.

[1] K. Hiruma, M. Yazawa, T. Katsuyama, K. Ogawa, K. Haraguchi, M. Koguchi, H. Kakibayashi, J. Appl. Phys. 1995, 77, 447-462.

[2] X. Duan, Y. Huang, Y. Cui, J. Wang, C. M. Lieber, Nature 2001, 409, 66-69.

[3] J. Wang, M. S. Gudiksen, X. Duan, Y. Cui, C. M. Lieber, Science 2001, 293, 1455-1457.

[4] R. S. Wagner, W. C. Ellis, Appl. Phys. Lett. 1964, 4, 89-90.

[5] M. Yazawa, M. Koguchi, A. Muto, K. Hiruma, Adv. Mater. 1993, 5, $577-580$.

[6] C.-C. Chen, C.-C. Yeh, Adv. Mater. 2000, 12, 738-741.

[7] Y. Wu, P. Yang, Chem. Mater. 2000, 12, 605-607.

[8] A. M. Morales, C. M. Lieber, Science 1998, 279, 208-211.

[9] X. Duan, C. M. Lieber, J. Am. Chem. Soc. 2000, 122, 188-189.

[10] W. S. Shi, Y. F. Zheng, N. Wang, C.-S. Lee, S.-T. Lee, Adv. Mater. 2001, 13, 591-594.

[11] A. D. Berry, R. J. Tonucci, M. Fatemi, Appl. Phys. Lett. 1996, 69, 2846-2848.

[12] W. Han, S. Fan, Q. Li, Y. Hu, Science 1997, 277, 1287-1289.

[13] H. W. Seo, S. Y. Bae, J. Park, H. Yang, S. Kim, Chem. Commun. 2002, 2564-2565.

[14] F. Wang, A. Dong, J. Sun, R. Tang, H. Yu, W. E. Buhro, Inorg. Chem. 2006, 45, 7511-7521.

[15] T. J. Trentler, K. M. Hickman, S. C. Goel, A. M. Viano, P. C. Gibbons, W. E. Buhro, Science 1995, 270, 1791-1794

[16] T. J. Trentler, S. C. Goel, K. M. Hickman, A. M. Viano, M. Y. Chiang, A. M. Beatty, P. C. Gibbons, W. E. Buhro, J. Am. Chem. Soc. 1997, 119, 2172-2181.
[17] H. Yu, J. Li, R. A. Loomis, L.-W. Wang, W. E. Buhro, Nat. Mater 2003, 2, 517-520.

[18] H. Yu, W. E. Buhro, Adv. Mater. 2003, 15, 416-419.

[19] F. Wang, H. Yu, J. Li, Q. Hang, D. Zemlyanov, P. C. Gibbons, L.-W. Wang, D. B. Janes, W. E. Buhro, J. Am. Chem. Soc. 2007, 129, $14327-14335$.

[20] F. Wang, W. E. Buhro, J. Am. Chem. Soc. 2007, 129, 14381-14387.

[21] J. Sun, W. E. Buhro, Angew. Chem. 2008, 120, 3259-3262; Angew. Chem. Int. Ed. 2008, 47, 3215-3218.

[22] J. Sun, L.-W. Wang, W. E. Buhro, J. Am. Chem. Soc. 2008, 130, 7997-8005.

[23] F. M. Davidson III, A. D. Schricker, R. J. Wiacek, B. A. Korgel, $A d v$. Mater. 2004, 16, 646-649.

[24] F. M. Davidson III, R. Wiacek, B. A. Korgel, Chem. Mater. 2005, 17, 230-233.

[25] J. Fang, K. L. Stokes, W. Zhou, W. Wang, J. Lin, Chem. Commun 2001, 1872-1873.

[26] A. Dong, H. Yu, F. Wang, W. E. Buhro, J. Am. Chem. Soc. 2008, 130, 5954-5961.

[27] B. A. Korgel, Nat. Mater. 2006, 5, 521-522.

[28] M. G. Jacko, S. J. W. Price, Can. J. Chem. 1964, 42, 1198-1205.

[29] M. C. Paputa, S. J. W. Price, Can. J. Chem. 1979, 57, 3178-3181.

[30] A. H. McDaniel, M. D. Allendorf, Chem. Mater. 2000, 12, 450-460.

[31] S. Xu, S. Kumar, T. Nann, J. Am. Chem. Soc. 2006, 128, 1054-1055.

[32] D. D. Fanfair, B. A. Korgel, Cryst. Growth Des. 2005, 5, 1971-1976.

[33] P. A. Fowell, C. T. Mortimer, J. Chem. Soc. 1958, 3734-3736.

[34] L. H. Long, J. F. Sackman, Trans. Faraday Soc. 1958, 54, 1797-1803.

[35] M. G. Jacko, S. J. W. Price, Can. J. Chem. 1963, 41, 1560-1567.

[36] W. D. Clark, S. J. W. Price, Can. J. Chem. 1968, 46, 1633-1634.

[37] Y.-F. Lin, W.-B. Jian, Nano Lett. 2008, 8, 3146-3150.

[38] Y.-F. Lin, W.-B. Jian, C. P. Wang, Y.-W. Suen, Z.-Y. Wu, F.-R. Chen, J.-J. Kai, Appl. Phys. Lett. 2007, 90, 223117/1-3.

[39] P. Dreszer, W. M. Chen, K. Seendripu, J. A. Wolk, W. Walukiewicz, B. W. Liang, C. W. Tu, E. R. Weber, Phys. Rev. B 1993, 47, 41114114.

[40] E. D. Minot, F. Kelkensberg, M. van Kouwen, J. A. van Dam, L. P. Kouwenhoven, V. Zwiller, M. T. Borgström, O. Wunnicke, M. A. Verheijen, E. P. A. M. Bakkers, Nano Lett. 2007, 7, 367-371.

[41] The electrical measurements were performed on straight portions of the GaP nanowires only and no planar twining faults in the selected length were involved.

[42] J.-R. Kim, B.-K. Kim, J.-O. Lee, J. Kim, H. J. Seo, C. J. Lee, J.-J. Kim, Nanotechnology 2004, 15, 1397-1400.

[43] The TOP was used for promoting the dissolution of $\mathrm{InMe}_{3}$.

Received: January 21, 2009 Published online: April 2, 2009 